\title{
Should Specific Values Be Embedded In The Internet Architecture?
}

\author{
Ian Brown ${ }^{1}$ \\ Oxford Internet Institute \\ Oxford, UK \\ ian.brown@oii.ox.ac.uk
}

\author{
David D. Clark ${ }^{2}$ \\ MIT CSAIL \\ Cambridge, MA, USA \\ ddc@csail.mit.edu
}

\author{
Dirk Trossen ${ }^{3}$ \\ Cambridge University \\ Cambridge, UK \\ dirk.trossen@cl.cam.ac.uk
}

\begin{abstract}
A fundamental disagreement in the Future Internet debate concerns architecture design and value. Should an architecture incorporate inherent values that have been widely accepted through societal debate or be adaptable once deployed to a wider range of stakeholder values put forward within an evolving societal context? This disagreement is reflected in many debates about the current and future Internet, such as over network neutrality. This article outlines the concrete viewpoints relating to this disagreement and describes its impact on the wider context of architecture design.
\end{abstract}

\section{Categories and Subject Descriptors}

C.2.1 [Computer-Communication Networks]: Network Architecture and Design - distributed networks, network communications.

General Terms

Design, Economics.

\section{Keywords}

Architecture, control points, value set

\section{INTRODUCTION}

The Internet, with its global scope, is embedded in a range of societies with differing norms, laws and customary expectations. Some societies are more open, some more controlled. Some give more power to the people, some to the state. Some give power to large corporate actors, others less so. Different countries have different laws that govern state surveillance, protection from search, and so on. Both within societies and across them, there is an ongoing process of debating these differences.

While in many respects societies often agree to disagree, the Universal Declaration of Human Rights (UDHR [1]) is a rare example of a set of values that is internationally recognized by the majority of societies.

Permission to make digital or hard copies of all or part of this work for personal or classroom use is granted without fee provided that copies are not made or distributed for profit or commercial advantage and that copies bear this notice and the full citation on the first page. To copy otherwise, to republish, to post on servers or to redistribute to lists, requires prior specific permission and/or a fee.

ACM ReArch 2010, November 30, 2010, Philadelphia, USA.

Copyright 2010 ACM 978-1-4503-0469-6/10/11...\$10.00.
The Internet of today is an artifact with a tremendous impact on societies and potentially their values. This societal aspect is likely to increase in any evolution of the Internet. It is therefore of no surprise that the societal contestation of value shaping increasingly enters the realm of the Internet in discussions about its governance and its design. Issues such as network neutrality or privacy are as much societal as they are design decisions. Therefore, it is imperative for the wider community of researchers not only to have clearly formulated viewpoints about these tussles but also to understand their impact on our work.

This paper contributes to this discussion by laying out the nature of the disagreement from a general architecture design perspective. It then describes two opposing viewpoints. Equally importantly, the paper addresses the potential impact that this discussion (and either viewpoint) might have on the wider issue of architecture design. It is important to understand that this paper is merely meant as the beginning of a much wider debate on architecture design and its relation to the society that we, as a community, have been influencing with our design choices in the past and with any choice we make in the future.

The methodology we use in this paper follows the recognition of initiatives like the EIFFEL think tank [2] that an agreement on the nature of particular disagreements is paramount for moving forward in any Future Internet effort, as outlined in [3]. This is reflected in the structure of the paper. Section 2 outlines the disagreement that is the basis for our discussions in this paper. Section 3 provides some background, outlining the importance of this debate for other areas of research. Section 4 then presents two opposing viewpoints in this disagreement, while we discuss the impact of this discussion in Section 5. We conclude the paper in Section 6.

\footnotetext{
${ }^{1}$ Ian Brown's research was supported by the Engineering and Physical Sciences Research Council under grant $\mathrm{EP} / \mathrm{G} 069557 / 1$.

${ }^{2}$ David Clark's research was supported by the Office of Naval Research under contract N00014-08-1-0898. The opinions contained are those of the author, and do not reflect the opinions of the ONR.

3 Dirk Trossen's research was supported by the $7^{\text {th }}$ Framework Programme of the EU under contract FP7INFSO-ICT 216173.
} 


\section{THE DISAGREEMENT}

As a first step, let us understand how the disagreement arises. The Internet is a relatively homogenous technical artifact defined by IETF standards. However, these standards allow some flexibility for different actors to exploit them in different ways in different contexts. The particulars of the Internet, with its points of flexibility and its points of rigidity, have come to be seen as expressing, or at least supporting, values such as open and unregulated communication. It less consistently supports one or another formulation of privacy. It does not do a robust job of protecting private communication, unless users exploit tools outside the architecture such as Tor [4].

These normative or value considerations may not have been explicitly on the minds of the original designers of the Internet, but should they be now? Should we design a future Internet to take a specific stand on issues such as private communication? Or should issues like this be left to the control of different societies-different jurisdictions with different norms and expectations? In other words:

Are there norms and values of global scope that

should intentionally be "designed in" (to the extent

possible) to an Internet, or should an Internet

architecture (to the extent possible) accommodate a

variable set of social values to be configured at run-

time in different contexts?

When we use the term architecture, we are focusing on the waist of the Internet hourglass (or any future equivalent of the current waist) - that set of fundamental assumptions and agreements that binds all the participants. In other words, our focus is that of the current Internet Protocol (and future possible replacements) as well as the concept of layering that comes with any choice of waist. Our disagreement is then whether a specific (pre-determined) value system should be integrated into the architecture ${ }^{4}$ or whether a varying set of values need to be accommodated by the architecture without breaking the main architectural foundation ${ }^{5}$.

A possible theoretical basis through which to look at this specific disagreement is that of enabling a pluralistic civil society [6][7]. Giving individuals and their voluntary associations (such as trade unions, campaign groups and faith-based organizations) certain freedoms can balance the power of the state. To the extent that we see the Internet as a global platform that supports an emerging civil society, we should then give fundamental priority to those tools that support voluntary and private association, with the understanding that what happens within those associations is not the business of the network to regulate. Our disagreement is then:

\footnotetext{
${ }^{4}$ A secondary consideration that follows this question is that of what this particular value system could be.

${ }^{5}$ This directly follows the debate on design for tussle [5], which argues for a design approach that accommodates occurring tussles at design as well as at runtime.
}

Should we support civil society by pre-determining the future Internet's main tools for implementation as an inherent value set (upon which a set of stakeholders found their actions) or leave its implementation open to a variety of choices that might evolve over time?

Before delving into the viewpoints addressing this disagreement, we provide some background to related discussions and potential disagreements.

\section{RELATION TO OTHER DEBATES}

The disagreement on embedding inherent values into the design of architectures has a deep connection to other past and ongoing debates in a variety of areas. We highlight a few here to indicate the potential impact that our discussion could have on these other areas:

- Openness vs. closedness of the Internet: The tussle between proponents for the openness of the Internet versus those who would like to establish a walled garden of well-defined 'Internet' services is a long running debate. Although mainly positioned as an economic battle between protectionism and market liberalism, it is deeply rooted in an argument about the 'right' value set to be applied in the running of the Internet. This has led to post-design extensions that are often at odds with the original design philosophy of the current Internet. Examples of these extensions are firewalls and deep-packet inspection equipment, among others. The Internet Society report on Internet futures [11] attempts to move this debate onto the level of value sets. Although proposing a very particular value set to pursue, its attempt to outline the varying value sets that are struggling for dominance in the global Internet shows the clear relation of this debate to our disagreement.

- Societal importance of the Internet: The Internet has long ago grown beyond a small research network into a world-wide backbone for many societal functions, including but not limited to government, health, education, leisure, entertainment, security, and many more. Many studies on the usage of the current Internet and the needs and requirements for the Future Internet are being conducted [12]. These studies show the varying value sets that the many stakeholders and users impose onto the current Internet. Designers of the Future Internet need to address this issue of varying value sets, either through limiting their realization or widely accommodating them in some design that still needs to be determined. Hence, our debate here is of paramount importance to the growing societal dimension of the Internet.

- Relation to other design debates: In Section 5, we assess various design choices from the angle of our disagreement. Each of these design choices (and the assessment of their options) is often the foundation for 
a disagreement in itself, ultimately driven by the considerations of the disagreement in this paper.

- New architectural approaches: A number of efforts, such as [13][14][15], have recently been initiated to investigate new architectural approaches to internetworking. Through their design, all of these efforts provide means to implement (certain) value sets within their architecture. Understanding which value sets are enabled and which ones possibly prohibited is crucial in evaluating the overall viability of these approaches to address identified needs and requirements of society as a whole. Hence, the very nature of our disagreement comes alive through these various design approaches. However, it is probably fair to say that none of these efforts address the potential impact that our discussion could have on the larger issue of architecture design - an impact that we outline in Section 5.

\section{THE VIEWPOINTS}

Having placed our disagreement in relation to other debates, we now turn to the presentation of the main viewpoints on this disagreement.

\subsection{Embedded Values are Key}

Some key, universal values - of which the UDHR is the most legitimate expression - should be baked into the architecture at design time and take some serious effort to overturn.

Design choices frequently have political consequences they shape the relative power of different groups in society. The Internet's end-to-end design principle, combined with user-controlled personal computers (Zittrain's "generative grid" [16]), has given users considerable autonomy. Against the strongest efforts of powerful governments, it has enabled the widespread use of communications protocols secure against signals intelligence agencies with billion-dollar annual budgets. The recording and movie industries complain that peer-to-peer filesharing software is being used to completely undermine their control of copyright works $^{6}$. Voice over IP tools are draining the telephony revenues of the very telecommunications companies whose connectivity they cannibalize.

Given the increasing impact of the Internet on everyday life around the globe, those designing its future architecture have a particular moral responsibility to consider the political impact of their choices:

"Consciously or not, deliberately or inadvertently, societies choose structures for technologies that influence how people are going to work, communicate, travel, consume, and so forth over a very long time. In the processes by which structuring decisions are made,

\footnotetext{
${ }^{6}$ An advocate for the opposing point of view would observe that this seems to assert criminality as a core normative value of the Internet.
}

different people are situated differently and possess unequal degrees of power as well as unequal levels of awareness." [17]

This is especially true of values such as free speech and privacy, which have a fundamental impact on individual autonomy and the distribution of power in societies. These values are protected in the constitutions of many countries, and have particular legitimacy when they have gained widespread democratic assent through processes such as participative constitutional conventions. Such exercises can be seen as a more abstract and representative version of the participatory design process, while dealing with "narrowly conceived self interests and hostile prejudices." [26]

Constitutional rights are frequently designed to protect less powerful groups in society against knee-jerk political reactions by majority groups. As such they are amendable only with widespread democratic consent over a considerable period of time. In this they have similar qualities to large-scale technical systems such as the Internet, where sunk costs and network effects can act as considerable barriers to architectural changes:

"Because choices tend to become strongly fixed in material equipment, economic investment, and social habit, the original flexibility vanishes for all practical purposes once the initial commitments are made. In that sense technological innovations are similar to legislative acts or political foundings that establish a framework for public order that will endure over many generations. For that reason the same careful attention one would give to the rules, roles, and relationships of politics must also be given to such things as the building of highways, the creation of television networks, and the tailoring of seemingly insignificant features on new machines. The issues that divide or unite people in society are settled not only in the institutions and practices of politics proper, but also, and less obviously, in tangible arrangements of steel and concrete, wires and semiconductors, nuts and bolts." [17]

In the absence of a worldwide constitution, can a set of globally agreed values be found for the global Internet? How does one judge whether specific values "are universal to all humans or pertinent only to groupings, whether nations, societies, cultures, religions, communities, or families"? [18]

While the emphasis on different values varies from state to state, all of the members of the United Nations have been able to find common agreement on the values proclaimed in the Universal Declaration of Human Rights. In looking for the most legitimate set of global values to embed in the future Internet architecture, the UDHR has the democratic assent of a significant fraction of the planet's population, through their elected representatives. Embedding values related to individual autonomy in the Internet architecture is one way for designers to support those that do not yet have the opportunity to elect their representatives, "even if they are not always able to prevail against the tide of countervailing forces." [18] 


\subsection{Catering to Variable Value Sets}

The architecture should accommodate variable value sets, i.e., it should be flexible enough so that important stakeholders, e.g., governments and local cultures, could instantiate their own values at runtime.

This point of view is not motivated by anything so fundamental as a rejection of the UDHR. The considerations are more pragmatic.

The rights expressed in the UDHR are not absolute. Even in countries that value free and unregulated speech there are limits, which vary by state. France prohibits hate speech, Thailand prohibits insults to the king, and the U.S. prohibits conspiratorial plotting to commit certain crimes. It is unrealistic to imagine that governments will simply abandon their right to enforce these prohibitions because the activity occurs over an Internet apparently designed to thwart them. The operative question is how they will proceed, and how that behavior will affect the Internet and its operation.

Technology is not the only tool in the tussle. All the authors of this paper would agree that the Internet, with its unfettered communication, has been a powerful force for open and free interaction and an empowerment of the individual and non-state actors. But this conclusion is not the same as hoping that at any instant, technology can trump law. State actors (and others) who find the behavior of the technology unwelcome and who have access to other tools of control (e.g., law and regulation) will fall back on those tools. A rigid Internet embedded in a varying regulatory context may be seen as a tool to escape enforcement, but at the cost of equating the Internet (in the minds of some) with a platform for criminality. Is this the best moral outcome, or should the network be able to conform to the law in all its variations - in authoritarian as well as democratic states?

Blunting the tools of enforcement is not always beneficial. The "theory of the blunt instrument" says that if you can blunt the tools of your antagonist in a tussle, you may be able to disable him. Thus, if all users of the Internet always encrypted everything they sent, there could be no wiretaps and no discrimination based on content. The only tool left to the regulator (e.g., the state) would be the blunt instrument of total disconnect. This theory is appealing to those who see the Internet as a vehicle for contention with state controls. And indeed, this theory is appealing and has much to recommend it. But (again) in the extreme, it suffers from two problems. First, we have seen states, faced with the only option of the blunt instrument, use it. When Google refused to continue to comply with the law of the land in China, China threatened to revoke their license to operate there, leaving Chinese users with the even-more regulated alternative of Baidu. Opinions may differ, but some argue that a little Google would be better for the
Chinese people than none ${ }^{7}$. Similarly, Burma took the step of disconnecting all international telecommunications links during the 2007 "Saffron Revolution", and several countries have blocked Facebook and YouTube. Are those countries and their citizens better off with that outcome than with half a loaf? Second, when law trumps technology, baking the "blunt instrument" outcome into the architecture may raise large complexities for actors required to comply with regulations. When France required that Yahoo block auctions of Nazi memorabilia to its citizens, Yahoo had to organize a table that identified (imperfectly) IP addresses of French users. Would it have been better or worse if the Internet made it easy to tell from what jurisdiction a connection came?

We should never seem to offer what we cannot. The open nature of the Internet will never be sufficient to provide total protection to those who want to evade state controls. We should never offer claims of protections we cannot provide, because the consequences may be serious. Activists in repressive countries face arrest, incarceration, and even death. We should be realistic about what the network can and cannot do.

\section{ASSESSING DESIGN OPTIONS}

While the general outcome of the debate in this paper is properly for the wider society to agree, the research community can contribute to this discussion by developing and providing a method to identify design choices that should be informed by this debate. Our discipline's objectives in evaluating design choices needs to widen from the narrow performance evaluation that many research efforts are still focusing on towards the larger socio-economic impact that some choices will have.

A starting point for this evaluation is the notion of control that is manifested in the various aspects of each design choice. The implementation of certain control points within a design (and its implementation) determines the ability to implement particular value sets of various stakeholders, defines the business models that economic actors can base their business on and outlines the spectrum for regulatory intervention that can (and needs to) be imposed on the system. Hence, understanding design choices is strongly connected to performing a deep control point analysis of a particular design (see [24] for an example of such analysis). While the results of this analysis are left for the wider society to take into consideration when converging towards an adoptable design for their needs, it is the scientific community that must develop the rigorous design framework that is the basis for such analysis.

\footnotetext{
${ }^{7}$ We recognize that this example has nothing to do with Internet architecture, but it illustrates the consequence of taking an absolute position. And this tussle is still ongoing. China may get some Google, in the end.
} 
Some examples that emphasize the need for a control point analysis, looking both at today and tomorrow, may help make this discussion more concrete.

Address assignment: Today IP addresses are allocated to institutions, such as ISPs and other providers. An alternative approach, advocated through the ITU by their Chinese delegation ${ }^{8}$, is to allocate future (IPv6) addresses to countries, which would then allocate them to providers within the countries. This approach would make it easier to map between an address and the home jurisdiction of that address, which would have a number of implications worthy of consideration, pro and con.

Address re-writing: The original design of the Internet was based on the assumption of a single global address space. Today, however, many packets carry addresses of intermediate nodes or middle-boxes (e.g., NAT devices), and those devices store enough internal state to rewrite the addresses as the packets pass through them. One of the implications of such rewriting is that in the region of the network where the packet carries only that intermediate address, one cannot determine the actual endpoint without obtaining the address mapping stored in the re-writer, which means that a policy-constrained step (e.g., conforming to legal requirements of a particular jurisdiction) is required to retrieve this information. In contrast, if the global IP address is revealed anywhere, then any observer along the path of the packet can capture the endpoint addresses.

Routing: Today, ISPs control the routing of packets, for a variety of reasons. The alternative of user-selected routes shifts the control from the ISP to the user, with a number of implications, including economics, pricing and cost recovery, capacity planning and connectivity engineering, robustness and resilience, and the power of choice to discipline competition [19]. Another approach, presented in [14], outlines a dedicated function for building interdomain routes in collaboration with parties in the process of information dissemination (i.e., publishers, subscribers as well as mediators and the involved transport ISPs).

DNS resolution: Some might say that the DNS is not part of the "narrow waist" of the Internet, but as a practical matter, essentially all services and applications depend on it. The DNS was designed to be robust to failure, but not with much attention to control point analysis. Today, the end-user normally just accepts the version of the DNS provided and controlled by his ISP, which may return answers that have been manipulated for various purposes. Even if the user attempts to use a DNS server of his choice, the requests may be redirected by his ISP. An alternative

\footnotetext{
${ }^{8}$ There is some disagreement as to whether the original proposal was for some or all IPv6 addresses to be allocated to countries. For a 2004 statement that makes clear that the proposal for only for some addresses to be allocated in this way, see http://www.itu.int/ITU-T/tsb-director/itut-wsis/files/zhaonetgov02.doc
}

design would allow the user to make a trustworthy connection to a DNS server of his choice, which would shift the balance of power away from the ISP and toward the user.

Virtualization: The waist of the (Internet) architecture has served as an enabler for many overlays that sit (architecturally) 'above' this waist. We see service overlays such as Tor [4], and applications themselves with their own models of freedom and control. In a sense, the IP layer provides a universal underlay for the many overlays that can be formed on top of it [8]. Some proposals for a future Internet have argued that the only narrow waist should be a virtualization layer, on top of which many competing examples will emerge to deal with the sorts of issues we discuss above: addressing, routing, naming and so on.

The concept of a universal underlay seems to express a value of total freedom. However, such a claim (and more concretely specific proposals for virtualization) should be subjected to a rigorous control point analysis. The control points will be found in the management architecture-what actors can set up an overlay, which policy drives the reachability of the overlay under what conditions, and so on. That interface could end up being very closed, and thus provide a tool for regulation, rather than for freedom. More philosophically, we could ask:

Should the waist be a universal 'underlay' for as many possible overlays as possible (as well as a universal 'overlay' for as many technology underlays as possible) or shall we prohibit (or at least complicate) specific overlays from happening?

Information dissemination: Another proposal for a future Internet architecture, very different from that of virtualization, is the idea that the "narrow waist" should focus on information naming and dissemination rather than packet transport between machines. One such proposal replaces the machine addresses in packets with named fragments of information [13]. This clever idea, however, if implemented at the level of the packet switch, seems to give the operator of the packet-level infrastructure a great deal of control over what information is delivered, and a great deal of visibility into which users are asking for which information. In another approach, labeling and scoping concepts are used, hiding the naming semantics from the infrastructure provider and therefore providing less leverage for unwanted control of traffic on that level [14]. Furthermore, the proposal separates the functions for information finding, determining a route between providers and consumers of the information and the final act of delivering the information across the network. Combined with label-based forwarding approaches [20], such separation allows for the creation of policy-based routes while enforcing policy-compliance on the level of individual forwarding networks, further curbing the operator's control. Only a thorough control point analysis of the design options within these schemes would allow for a critical assessment of what values they could express. 


\section{CONCLUSIONS}

There are many impacts that this discussion could have on the work of the wider research community. A crucial issue that we can take away from our discussion so far is that a deep understanding of design choices, their impact on society at large and their implementation in design processes is a central issue that underlies this disagreement.

There is a tension between our community's desire, on the one hand, to be more methodical in our design, and the desire, on the other hand, to fold in these larger considerations that are difficult to quantify. The need for a more rigorous design framework has been recognized in many research efforts. For instance, work from Touch [9] and Day [21] introduces a scientific approach to the assembly of distributed systems while the field of network economics is increasingly introducing economic models into the network area [22]. Even processes observed in evolution theory are applied on the level of system design [23]. The approach presented in [24] outlines a control point analysis for large-scale functions within a networked system with the example of large-scale rendezvous and discovery. The work is folded into a system dynamics framework [25], resulting in analytical models for evaluating socio-economic outcomes under a variety of scenarios. This approach, among others, potentially leads to a greater understanding of what one could call Internet Science, which must somehow take into account not only issues of technical optimization but issues of making choices in the larger social and legal context in which the Internet is embedded.

\section{REFERENCES}

[1] U.N., "Universal Declaration of Human Rights", available at http://www.un.org/en/documents/udhr/, 2010

[2] EIFFEL think tank, available at http://www.fp7-eiffel.eu, 2010

[3] D. Trossen (ed), "Invigorating the Future Internet Debate", ACM SIGCOMM Computer Communications Review, October 2009

[4] Tor project, "Tor: anonymity online", available at http://www.torproject.org/, 2010

[5] D. Clark, J. Wroclawski, K. R. Sollins, R. Braden, "Tussle in Cyberspace: Defining Tomorrow's Internet," in IEEE/ACM Transactions on Networking, Vol. 13, No. 3, 2005

[6] John Keane, "Global Civil Society?", Cambridge University Press, 2003

[7] John R. Hall (Editor), "Civil Society: Theory, History, Comparison”, Polity Press, 1995

[8] D. Clark, B. Lehr, S. Bauer, P. Faratin, R. Sami, J. Wroclawski, "Overlay Networks and the Future of the
Internet", Communications \& Strategies, no. 63 , p. 1, 3rd quarter 2006

[9] J. Touch, Y. Wang, V. Pingali, "A Recursive Network Architecture", ISI Technical Report ISI-TR-2006-626, October 2006

[10] N. Fotiou, G. C. Polyzos, D. Trossen, "Illustrating a PublishSubscribe Internet Architecture", Proceedings of Future Internet Architectures: New Trends in Service Architectures (2nd Euro-NF Workshop), September 2009

[11] Internet Society, "Internet Futures Scenarios", Technical report of the ISOC, October 2009

[12] S. Forge, K. Guevara, L. Srivastava, C. Blackman, J. Cave, "Interim Report: Towards a Future Internet", Oxford Internet Institute, May 2010

[13] V. Jacobson, D. K. Smetters, J. D. Thornton, M. Plass, N. Briggs, R. L. Braynard, "Networking Named Content", ACM CoNext, December 2009

[14] D. Trossen, M. Sarela, K. Sollins, "Arguments for an Information-Centric Internetworking Architecture", ACM Computer Communication Review 40(2), pp. 27-33, 2010

[15] T. Koponen et al., "A Data-Oriented Network Architecture", ACM SIGCOMM, 2007

[16] J. Zittrain, "The Future of the Internet - And How to Stop It", Yale University Press, 2008

[17] L. Winner, "Do Artifacts Have Politics?" Daedalus 109, pp. 121-36, 1980

[18] M. Flanagan, D. Howe, H. Nissenbaum, "Embodying Values in Technology: Theory and Practice", In Information Technology and Moral Philosophy. J. van den Hoven and J. Weckert (eds.) Cambridge University Press, pp. 322-353, 2008

[19] K. Lakshminarayanan, I. Stoica, S. Shenker, "Routing as a Service”, UCB/CSD-04-1327, UC Berkeley, 2004

[20] P. Jokela, A. Zahemszky, S. Arianfar, P. Nikander, C. Esteve, "LIPSIN: Line speed Publish/Subscribe InterNetworking", ACM SIGCOMM, August 2009

[21] J. Day, "Patterns in Network Architecture: A Return to Fundamentals", Pearson Education, 2008

[22] C. Vaishnav, "Does Technology Disruption Always Mean Industry Disruption?", Conference of System Dynamics Society, 2008

[23] C. Dovrolis, "What would Darwin think about clean-slate architectures?", ACM SIGCOMM Computer Communication Review 38 (1), pp. 29-34, 2008

[24] J. Riihijärvi, D. Trossen, et al, "First report on quantitative and qualitative architecture validation", Deliverable D4.2 of the PSIRP project, 2009

[25] J. D. Sterman, "Business Dynamics: Systems Thinking and Modeling for a Complex World", McGraw-Hill Higher Education, Boston, 2000

[26] B. Friedman, P. Kahn, A. Borning, "Value Sensitive Design: Theory and Methods", UW CSE Technical Report 02-12-01, 2002 